\title{
El consumo de tabaco en los hogares en México: resultados de la Encuesta de Ingresos y Gastos de los Hogares, $1984-2000$
}

\author{
Luis A lonso Vázquez-Segovia, Lic Soc, ${ }^{(1)}$ Sergio Sesma-Vázquez, Mtro en Economía de la Salud, ${ }^{(2)}$ \\ Mauricio Hernández-Avila,PhD. ${ }^{(3)}$
}

\section{Vázquez-Segovia LA, Sesma-Vázquez S, Hernández-Avila M. El consumo de tabaco en los hogares en México: resultados de la Encuesta de Ingresos y Gastos de los Hogares, 1984-2000. Salud Publica Mex 2002;44 supl 1:S76-S81. El texto completo en inglés de este artículo está disponible en: http://www.insp.mx/salud/index.html}

\section{Resumen}

Objetivo. Analizar algunos de los determinantes del consumo de tabaco en los hogares en México. Material y métodos Los datos provienen de la Encuesta $\mathrm{N}$ acional de Ingresos y $G$ astos de los H ogares (1984-2000). El análisis se realizó en dos periodos: 1984-1992 y 1994-2000. Las posibilidades de consumo de tabaco en hogares (PCTH) fueron estimadas mediante un modelo de regresión logística. $\mathbf{R e}$ sultados La PCTH durante el primer periodo fue superior a dos veces a partir del decil $6(\mathrm{RM}=2.03, \mathrm{IC} 95 \%$, 2.03-2.04) y durante el segundo periodo a partir del decil 8 (RM $=2.06$, IC 95\%, 2.05-2.07). Los hogares del decil 1 gastaron en promedio de 9 a 22\% de sus ingresos en tabaco; casi todos los hogares del decil 10 , solamente $1 \%$. Conclusiones Los ho gares con mayores ingresos consumieron más tabaco que los hogares de menores ingresos, sin embargo, los hogares de más bajos ingresos destinaron un porcentaje más importante de éstos al consumo de tabaco. El texto completo en inglés de este artículo está disponible en: http://www.insp.mx/salud/index.html

Palabras clave: consumo de tabaco; gasto en tabaco; tabaquismo; México

\author{
Vázquez-Segovia LA, Sesma-Vázquez S, \\ Hernández-Avila M. \\ Tobacco consumption in Mexican households: \\ results from the National Household Income \\ and Expenditure Survey, 1984-2000. \\ Salud Publica Mex 2002;44 suppl 1:S76-S81. \\ The English version of this paper \\ is available at: http://www.insp.mx/salud/index.html
}

\begin{abstract}
A bstract
Objective. To analyze selected determinants of tobacco consumption in Mexican homes. Material and Methods. D ata from the $\mathrm{N}$ ational $\mathrm{H}$ ousehold Income and Expenditure Survey, 1984-2000, were analyzed.The analysis was conducted for the years 1984-1992 and 1994-2000. The likelihood of tobacco consumption in households was estimated with a logistic regression model. Results Tobacco consumption during the first period was two times more likely from decile six (OR=2.03, 95\%, Cl: 2.03-2.04) and during the second period from decile eight $(\mathrm{O} R=2.06,95 \%, \mathrm{Cl}: 2.05-2.07)$. Households from decile one spent on average $9-22 \%$ of their income on tobacco; almost all of the households from decile ten spent only $1 \%$ of their income on tobacco. Conclusions. Households with higher income consumed more tobacco than households with low income; nevertheless, households with the lowest income devoted a greater proportion of income to to bacco consumption. The English version of this paper is available at: http://www.insp.mx/salud/index.html
\end{abstract}

Key words: tobacco comsumption; tobacco expenditure; smoking; Mexico

(1) Centro de Investigaciones en Salud Poblacional, Instituto N acional de Salud Pública, México.

(2) Dirección General de Información y Evaluación del Desempeño, Secretaría de Salud, México

(3) Centro de Investigaciones en Salud Poblacional, Instituto N acional de Salud Pública, México.

Fecha de recibido: 9 de enero de 2002 - Fecha de aprobado: 26 de abril de 2002

Solicitud de sobretiros: Lic. Luis Alonso Vázquez-Segovia. Centro de Investigaciones en Salud Poblacional. Instituto N acional de Salud Pública,Avenida Universidad 655, Colonia Santa María A huacatitlán, 62508, Cuernavaca, Morelos, México.

Correo electrónico: lalonsov@ correo.insp.mx 
E n México se han realizado diversos estudios para establecer la prevalencia de tabaquismo en la población general con representación en el ámbito nacional, tales como la Encuesta Nacional de Salud (ENSA) $1986^{1}$ y $2000{ }^{2}$ y la Encuesta Nacional de Adicciones (ENA) 1988, 1993 y 1998. 3-5 Otras encuestas también han tenido cobertura nacional pero dirigidas a poblaciones específicas, como la Encuesta Nacional sobre Uso de Drogas entre la Comunidad Escolar, y múltiples encuestas en diversas entidades y centros de educación desde la década de los setenta. ${ }^{6}$

En este trabajo presentamos un análisis del consumo de cigarrillos en los hogares, reportado en la Encuesta Nacional de Ingresos y Gastos de los Hogares (ENIGH) realizadas en 1984, 1989, 1992, 1994, 1996, 1998 y $2000,{ }^{7-9}$ al que nos referiremos a partir de ahora con la denominación genérica de tabaco. La ENIGH registró el tabaco en dos tipos más de presentaciones - puros y tabaco picado o en hoja- que no fueron incluidos en este trabajo. La ENIGH nos permitirá observar la evolución que ha seguido el consumo de tabaco en México y su relación con algunos de sus determinantes, como el nivel de instrucción y el sexo del jefe del hogar, el nivel de ingresos de los hogares, $y$ el consumo de bebidas alcohólicas.

\section{Material y métodos}

La unidad de muestreo fue la vivienda, y la unidad de observación el hogar. Todas las encuestas se llevaron a cabo durante el tercer trimestre del año, con excepción de la ENIGH 1994 realizada entre septiembre y diciembre. La ENIGH tiene una cobertura geográfica nacional para localidades de más de 2500 habitantes y de menos de 2500 . El diseño de la muestra fue de tipo probabilístico, polietápico, estratificado y por conglomerados. El número de viviendas en muestra fue de 5264 en 1984; 14082 en 1989; 12411 en 1992; 15385 en 1994; 16875 en 1996, 12775 en 1998 y 14781 en $2000 .{ }^{10-17}$ La tasa de no respuesta ha variado entre 13 y $18 \%$. Para recolectar la información se utilizó un cuestionario básico y un cuadernillo de gastos. Es importante señalar que entre 1984 y 1992 ambos instrumentos contaban con un apartado específico para la captación del gasto en tabaco, sin embargo, a partir de 1994 fue eliminado para ser incluido en uno más general en el que se captaron alimentos, bebidas y tabaco. ${ }^{9,18}$ Por tal razón los resultados serán presentados en dos periodos: 1984-1992 y 1994-2000.

La variable dependiente fue el hogar con consumo de tabaco que registra si se consumió o no. Como variables independientes utilizamos el nivel de instrucción y el sexo del jefe del hogar, el ingreso corrien- te monetario trimestral total del hogar, y el consumo de bebidas alcohólicas y el año de realización de la encuesta. Nivel de instrucción registra el año de estudios más alto aprobado por el jefe del hogar. Esta variable fue recodificada en cuatro categorías: 1 . Sin escolaridad y primaria. 2. Secundaria. 3. Bachillerato o vocacional. 4. Licenciatura y posgrado. La variable sexo clasifica a los jefes del hogar en mujeres y hombres. Los jefes del hogar ausentes fueron excluidos del análisis.

El ingreso corriente monetario trimestral total del hogar, es decir, la cantidad de dinero que recibe un miembro del hogar por su trabajo, por utilidades, por rendimientos e indemnizaciones y transferencias corrientes, ${ }^{*}$ se obtuvo sumando los ingresos de todos los miembros del hogar. Los hogares en los que no se declararon ingresos fueron excluidos del análisis. El porcentaje del ingreso trimestral gastado en tabaco se obtuvo dividiendo el gasto trimestral en tabaco entre el ingreso trimestral total del hogar. Ambos, ingresos y gastos, fueron deflactados a pesos constantes de 1994. Consumo de bebidas alcohólicas es una variable dicotómica que clasifica a los hogares en aquellos que no adquirieron este tipo de bebidas de los que sí lo hicieron.

El procesamiento y análisis de datos se realizó utilizando el Paquete Estadístico para las Ciencias Sociales (SPPS ver. 10.1). En cuanto al análisis multivariado se corrió un modelo de regresión logística para cada periodo.

\section{Resultados}

En general la tendencia de consumo de tabaco en hogares experimentó un descenso, pasando de 21 a $9 \%$. El nivel de ingresos incrementó la PCTH (cuadro I). En efecto, entre 1984 y 1992 los hogares ubicados a partir del decil 6 en adelante tuvieron dos veces más posibilidades de consumir tabaco con respecto a los hogares del primer decil, muy cercana a tres veces en el décimo decil. En el segundo periodo la posibilidad de consumir tabaco dos veces y más con respecto al grupo de referencia, ocurrió a partir del decil 7, sin llegar al nivel de consumo alcanzado en el decil 10 del primer periodo. Este hecho evidenció un descenso en el consumo de los hogares de más altos ingresos.

\footnotetext{
* Bajo este rubro se clasifican las remuneraciones al trabajo (sueldos, salarios y horas extras, etcétera); la renta empresarial (provenientes de un negocio no agropecuario o agropecuario), renta de la propiedad (alquileres de terrenos, casas, edificios, locales, etcétera); los ingresos netos de cooperativas; las transferencias (percepciones que no constituyen el pago por trabajos realizados); y otros ingresos. ${ }^{9}$
} 


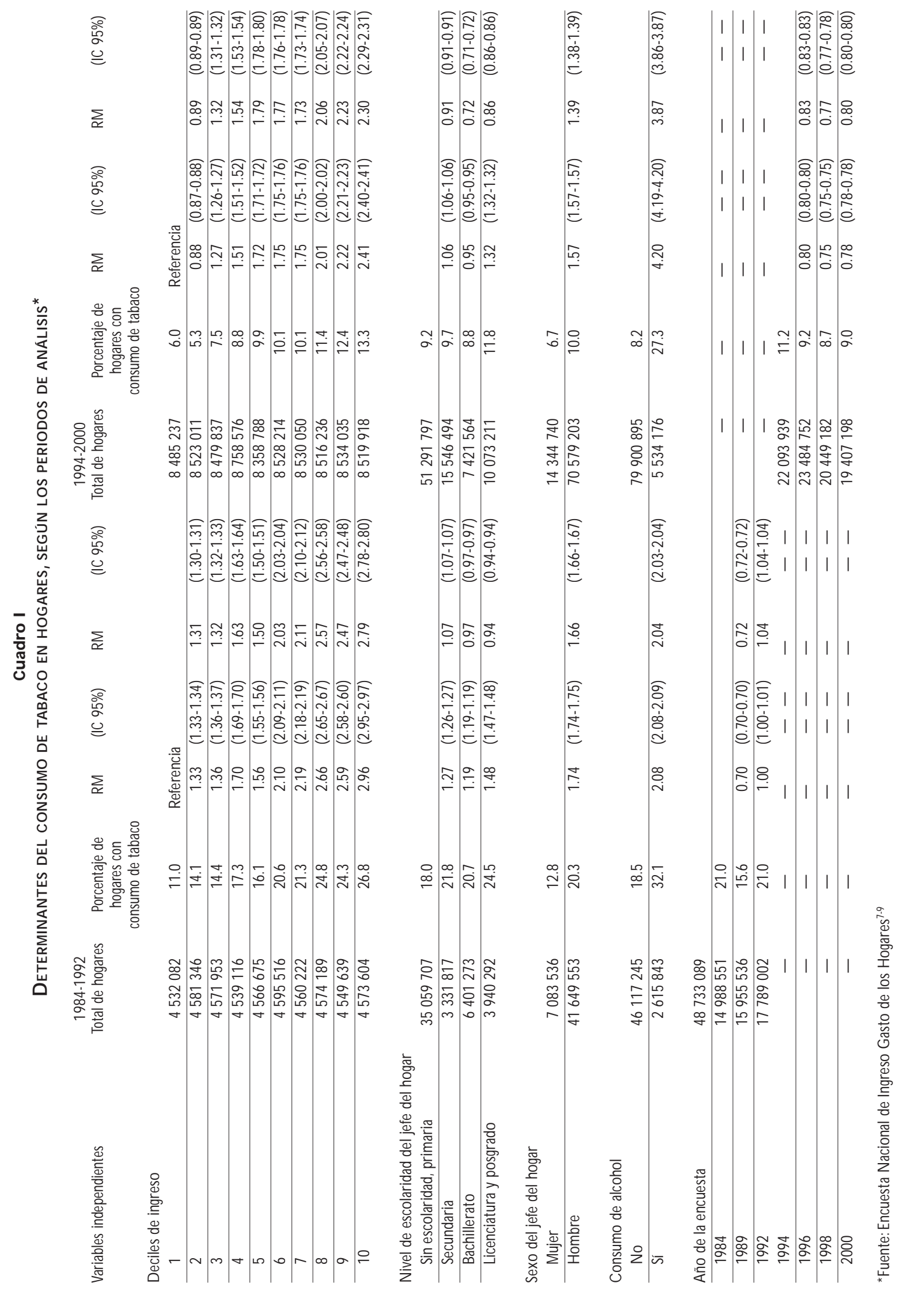


En ambos periodos observamos que cuando la posición de jefe del hogar estaba ocupada por un hombre, la PCTH fue más alta que cuando una mujer la ocupaba. En cuanto al nivel de instrucción del jefe del hogar, ésta tuvo una relación significativa en el primer periodo, en los hogares en los que el jefe del hogar tuvo un nivel de instrucción de secundaria.

El consumo de bebidas alcohólicas tuvo una de las contribuciones más importantes. Efectivamente, mientras en el periodo 1984-1992 la posibilidad de consumir tabaco fue de más de dos veces respecto a los hogares en los que no se consumieron bebidas alcohólicas, en el siguiente periodo dicha posibilidad ascendió a casi cuatro veces.

Un hecho relevante es que en promedio, para todos los años, los hogares de más bajos ingresos (decil 1) gastaron en tabaco entre 9 y $22 \%$ de sus ingresos. En el extremo contrario, en los hogares de más altos ingresos (decil 10) el gasto promedio en tabaco representó el 1\% de sus ingresos (figura 1). En el decil 2 el porcentaje promedio del gasto en tabaco estuvo entre 5 y $7 \%$, y va descendiendo conforme aumenta el nivel de ingreso del hogar.

\section{Discusión}

Existen notorias diferencias en los resultados de las diversas encuestas llevadas a cabo en nuestro país. La ENSA de 1986 mostró una prevalencia de 17.5\%. ${ }^{1} \mathrm{La}$ ENSA 2000, con representación en los ámbitos urbano y rural, mostró que la prevalencia en la población general (10 años de edad en adelante) fue de $17.8 \% .^{2}$ Los resultados de la ENA de 1988 mostraron que la prevalencia de fumadores entre la población urbana, de 12 a
65 años de edad fue de $25.8 \%,{ }^{3}$ la de 1993 fue de $25.1 \%$, y la de 1998 de $27.7 \% .{ }^{6}$ Diferencias que en el caso de la ENIGH pudieron haber estado influenciadas por las modificaciones en los instrumentos de captación de información efectuadas en 1994, y porque además no fue una encuesta diseñada para el estudio específico de adicciones, generando el subregistro del consumo de tabaco. Otro punto de referencia es la producción de cigarrillos que, de acuerdo con la Encuesta Industrial Mensual, experimentó en el periodo 1980-1996 un crecimiento, pasando de 2726000000 a 2995354000 de cajetillas. ${ }^{19,20}$

Como señala Basáñez, la década de los ochenta en México estuvo marcada por las crisis de 1982 y 1987,,21 al igual que la década de los noventa con la crisis de 1994. En este contexto el ingreso ha mostrado y continúa mostrando una distribución desigual ${ }^{22}$ y una tendencia al crecimiento de dicha desigualdad. ${ }^{23}$ En el estudio sobre Tabaquismo y Salud en las Américas se indicaba que el descenso en el consumo de tabaco ocurrido durante la década de los ochenta parecía estar más relacionado con el deterioro económico de los hogares que con una cuestión de salud. ${ }^{24}$ Con el fin de controlar la inflación, en 1990, el precio de los cigarrillos no se incrementó y al mismo tiempo fue considerado como parte de la canasta básica. Ambas medidas tuvieron como finalidad propiciar el crecimiento del empleo en la industria tabacalera y evitar afectar la producción. $^{25}$

El estudio de Kaplan y Lynch señala que la prevalencia de tabaquismo en las décadas de los ochenta y noventa, en los países en desarrollo, estaba más asociada con el nivel socioeconómico alto, ${ }^{26}$ tal como es posible observar en los resultados encontrados en la

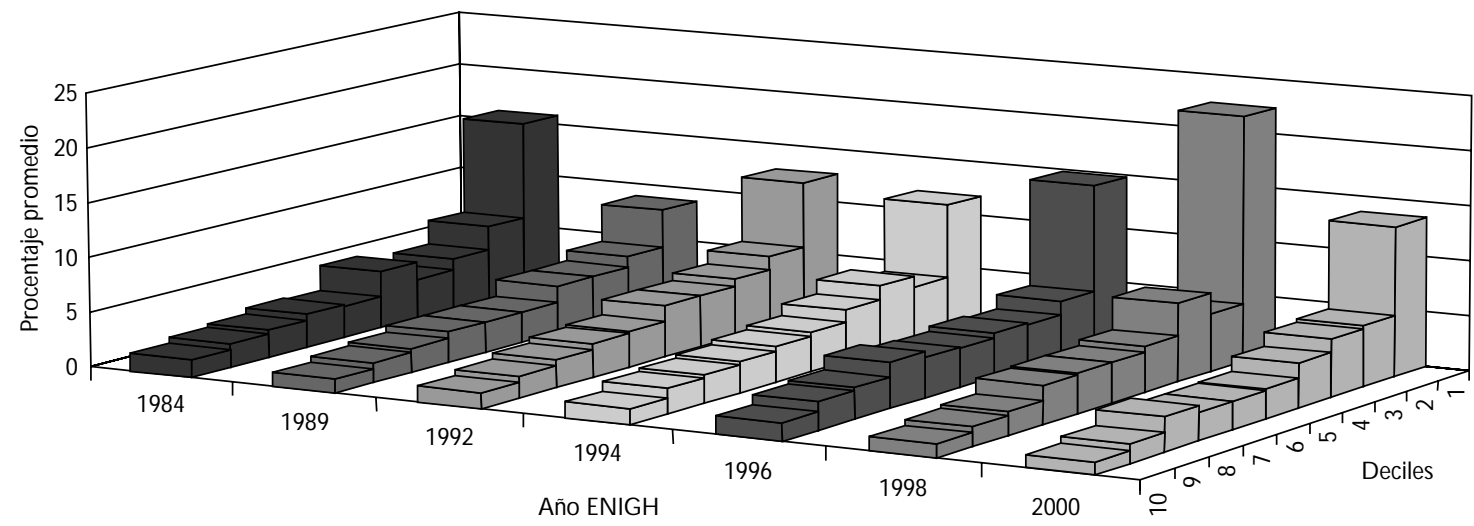

Figura 1. Encuesta Nacional de Ingreso Gasto de los Hogares. ${ }^{7-9}$ 
ENIGH, en los que encontramos que el consumo se incrementó conforme aumentaba el nivel de ingresos del hogar.

Los resultados de la Encuesta Nacional sobre Uso de Drogas entre la Comunidad Escolar, realizada entre la población estudiantil de enseñanza media y media superior, indicaron que $10 \%$ fumaron el mes anterior al estudio, y 3\% diario o casi diario. El 8\% de los hombres fumó el mes anterior al estudio en comparación con 3\% de mujeres. ${ }^{5}$ El estudio de Rojas y colaboradores, utilizando la misma encuesta pero centrada en el análisis de la población de los estados de la zona fronteriza del norte del país, mostró que el consumo de alcohol en el mes anterior al estudio (11 a 21\%) era más elevado que el de tabaco (8 a $11 \%$ ). El estudio mostró que las mujeres consumieron menos bebidas alcohólicas que los hombres: 1.6 contra 5.5\% se embriagaron de una a tres veces al mes en el último año anterior a la encuesta. ${ }^{27}$

\section{Conclusiones}

El consumo de tabaco en el quinquenio 1996-2000 muestra signos claros de haberse estabilizado. Es necesario continuar con las actividades de prevención y control, sobre todo si tomamos en cuenta que la ENA señala que el tabaquismo en las áreas urbanas ha experimentado un crecimiento.

Llama la atención que los hogares donde el jefe del hogar es hombre el consumo haya descendido, lo que puede significar que el alcance que la publicidad de la industria tabacalera está teniendo entre las mujeres jefas de hogar de ingresos más altos esté logrando el objetivo de captar a este sector de la población.

El vínculo tabaco-alcohol es sin duda de gran relevancia debido a las consecuencias que su consumo puede tener en la salud de la población. En el caso del tabaco los efectos en la morbilidad y mortalidad puede ser: enfermedad pulmonar obstructiva crónica, enfisema pulmonar, cáncer de pulmón, enfermedad isquémica cardiaca, muerte prematura, etcétera. En cuanto al consumo de alcohol también está asociado con enfermedades crónicas (hígado y cirrosis), la dependencia, los conflictos familiares, peleas o riñas, accidentes, etcétera.

Finalmente, y es sin duda una de las cuestiones más relevantes, el hecho de que la población de los hogares de más bajos ingresos utilicen una parte importante de sus recursos en tabaco exige que se hagan más análisis para profundizar en aspectos como alimentación y tabaquismo.

\section{Agradecimientos}

Agradecemos a los maestros Raydel Valdés, Luisa María Sánchez Zamorano, Julio César Campuzano y al doctor Francisco López Antuñano, del Centro de Investigaciones en Salud Poblacional; a los maestros Magali Cuadra Hernández, Daniel Hernández Rosete, y Margarita Márquez Serrano, del Centro de Investigaciones en Sistemas de Salud, todos ellos del Instituto Nacional de Salud Pública, los comentarios críticos al documento.

\section{Referencias}

1. Secretaría de Salud. Encuesta N acional de Salud. México: Secretaría de Salud, 1988.

2. Instituto $\mathrm{N}$ acional de Salud Pública.A nálisis de prevalencias de tabaquismo. Encuesta N acional de Salud 2000.

3. Secretaría de Salud. Encuesta Nacional de Adicciones 1998. México: Secretaría de Salud, 2002. Disco compacto.

4. Kuri-Morales P, Revuelta-HerreraA,Tapia-C onyer R. Prevalencia del consumo de tabaco en México. Gac Med Mex 1995;131:605-607.

5.Tapia-C onyer R, C ravioto P, Medina-Mora ME, Hoy MJ, Kuri P. Encuestas nacionales de adicciones 1988, 1993, 1998. En: Peña-C orona Gutiérrez MP, Kuri-Morales P,Tapia-Conyer R, comp. El consumo de tabaco en México y Encuesta N acional de Adicciones 1998 (Tabaco). México: Secretaría de Salud, 2000.

6.Villatoro J, Medina-Mora ME, Blanco J,Villa J, Martínez M, Fleiz C. Encuesta de consumo de drogas en estudiantes II. Disco compacto. México, D.F.: Secretaría de Educación Pública, Instituto Mexicano de Psiquiatría, 2001. 7. Instituto $\mathrm{N}$ acional de Estadística, Geografía e Informática. Encuesta $\mathrm{N}$ acional de Ingreso $\mathrm{G}$ asto de los Hogares. Sistema de Tabulados Básicos de la EN IG H 1984, 1989 y 1992, y Sistema de consulta a bases de datos de la EN IGH 1984, 1989 y 1992. México, D.F.: IN EGI, 1994;vol. I y II. (D isco compacto 1 y 2).

8. Instituto $\mathrm{N}$ acional de Estadística, Geografía e Informática. Encuesta $\mathrm{N}$ acional de Ingresos y Gastos de los Hogares. México, D.F.: IN EGI, 2000. (Disco Compacto).

9. Instituto $\mathrm{N}$ acional de Estadística, G eografía e Informática. Encuesta $\mathrm{N}$ acional de Ingresos y Gastos de los Hogares 2000. México, D.F.: IN EGI, 2001. (Disco Compacto).

10. Instituto $\mathrm{N}$ acional de Estadística, Geografía e Informática. Encuesta $\mathrm{N}$ acional de Ingresos y Gastos de los Hogares 1996. Documento Metodológico. México, D.F.: IN EGI, 1999:133-142.

11. Instituto N acional de Estadística, Geografía e Informática. Encuesta $\mathrm{N}$ acional de Ingresos y $\mathrm{G}$ astos de los Hogares. Cuarto trimestre de 1984. México, D.F.: IN EGI, 1989:163.

12. Instituto Nacional de Estadística, Geografía e Informática. Encuesta $\mathrm{N}$ acional de Ingresos y $\mathrm{G}$ astos de los Hogares 1989. Características de la vivienda por niveles de ingreso de su(s) hogar(es). México, D.F.: IN EGI, 1992:125.

13. Instituto Nacional de Estadística, Geografía e Informática. Encuesta $\mathrm{N}$ acional de Ingresos y $\mathrm{G}$ astos de los Hogares 1992. México, D.F.: IN EGI, 1993:191.

14. Instituto N acional de Estadística, G eografía e Informática. EN IGH 1994. Encuesta $\mathrm{N}$ acional de Ingresos y $\mathrm{G}$ astos de los Hogares. México, D.F.: IN EGI, 1995:307. 
15. Instituto Nacional de Estadística, Geografía e Informática. Encuesta $\mathrm{N}$ acional de Ingresos y $\mathrm{G}$ astos de los Hogares 1996. México, D.F.: IN EGI, 1998:757.

16. Instituto N acional de Estadística, Geografía e Informática. EN IGH 1994. Encuesta N acional de Ingresos y Gastos de los Hogares. EN IGH, 1998. México, D.F.: IN EGI, 2000:552.

17. Instituto N acional de Estadística, G eografía e Informática. EN IGH 1994. Encuesta N acional de Ingresos y Gastos de los Hogares 2000. EN IGH 2000. México, D.F.: IN EGI, 2001:516.

18. Instituto $N$ acional de Estadística, Geografía e Informática. Encuesta $\mathrm{N}$ acional de Ingreso-G asto de los Hogares, 1983-1984. Cuadernillo Auxiliar de Gastos. México, D.F.: IN EGI, s.f.:24. Encuesta N acional de Ingreso$G$ asto de los Hogares, 1989. Cuadernillo Auxiliar de Gastos. México, D.F.: IN EGI, 1989:24.

19. Meneses-G onzález F, Márquez-Serrano M, Sepúlveda-A mor J, Hernández-Avila M. Gasto en tabaco. En: Peña-Corona Gutiérrez MP, Kuri-Morales $P$,Tapia-C onyer $R$, comp. El consumo de tabaco en México y Encuesta $N$ acional de Adicciones 1998 (Tabaco). México, D.F.: Secretaría de Salud, 2000:33-50.

20. Instituto N acional de Estadística, Geografía e Informática. Banco de Información Económica. Encuesta Industrial Mensual. México, D.F.: IN EG I. http://dgcnesyp.inegi.gob.mx/BIE.HTML-SSI.

21. Basáñez M. El pulso de los sexenios. 20 años de crisis en México, D.F.: 2a edición. México, D.F.: Siglo XXI, 1991:17.
22. Vidal G. Grandes empresas, economía y poder en México. México: Universidad Autónoma Metropolitana y Plaza y Valdés, 2000.

23. A guilar-Gutiérrez G. D esigualdad y pobreza en México. ¿Son inevitables? México, D.F.: Universidad N acional Autónoma de México, Instituto de Investigaciones Económicas y Sociales, Instituto Politécnico $\mathrm{N}$ acional, Centro de Investigaciones Económicas, Administrativas y Sociales, Miguel Angel Porrúa, 2000.

24. Departamento de Salud y Servicios Sociales de los Estados Unidos de América, Centro para el Control de Enfermedades, O rganización Panamericana de la Salud, 0 rganización Mundial de la Salud.Tabaquismo y salud en las américas. A tlanta, G eorgia: D epartamento de Salud y Servicios Sociales de los Estados Unidos de América, Centro para el Control de Enfermedades, 0 rganización Panamericana de la Salud, 0 rganización Mundial de la Salud,1992.

25. Poder Ejecutivo. Diario 0 ficial de la Federación. México, D.F.: Poder Ejecutivo, 1990; tomo CDXL(10):19.

26. Kaplan GA, Lynch JW. Socioeconomic considerations in the primordial prevention of cardiovascular disease. Preventive Medicine 1999;29: S30-S35.

27. Rojas E, Medina-Mora ME, G alván-Reyes J, Juárez-G arcía F, C arreñoGarcía S, Berenzon-Gorn S et al. "Prevalencia de consumo de drogas entre estudiantes de la zona fronteriza". En: O rtiz A, Trejo E, comp. Las adicciones: un enfoque multidisciplinario. México, D.F.: Secretaría de Salud, Consejo N acional Contra las Adicciones, AMPEA, 1993:97-105. 